\title{
Campus numériques : des tendances innovantes au croisement d'enjeux pédagogiques, industriels et institutionnels
}

Virtual campuses: innovation tendencies build at the intersection of

pedagogical, industrial and institutional needs

\section{Alexandra Bal et Yolande Combès}

\section{OpenEdition}

Journals

Édition électronique

URL : http://journals.openedition.org/edc/608

DOI : $10.4000 /$ edc. 608

ISSN : 2101-0366

Éditeur

Université Lille-3

\section{Édition imprimée}

Date de publication : 1 décembre 2007

Pagination : 151-171

ISBN : 978-2-9514961-8-7

ISSN : $1270-6841$

\section{Référence électronique}

Alexandra Bal et Yolande Combès, « Campus numériques : des tendances innovantes au croisement d'enjeux pédagogiques, industriels et institutionnels », Études de communication [En ligne], Numéro spécial | 2007, mis en ligne le 01 octobre 2009, consulté le 20 avril 2019. URL : http:// journals.openedition.org/edc/608 ; DOI : 10.4000/edc.608

Ce document a été généré automatiquement le 20 avril 2019

(c) Tous droits réservés 


\title{
Campus numériques : des tendances innovantes au croisement d'enjeux pédagogiques, industriels et institutionnels
}

\author{
Virtual campuses: innovation tendencies build at the intersection of \\ pedagogical, industrial and institutional needs
}

Alexandra Bal et Yolande Combès

1 Les recherches conduites dans le cadre de l'ERTe se situent à notre sens à un moment clé où devient plus perceptible l'hésitation entre différentes façons d'utiliser les technologies d'information et de communication dans la sphère universitaire.

2 Ce sont des enseignants participant à des mouvements pédagogiques contemporains qui se saisissent assez généralement des Tice au départ, à l'appui d'un projet de rénovation des façons d'enseigner et d'apprendre. Comme l'explique Odile Coppey, chef du projet CampusCultura : «pour un chef de projet, la conviction pédagogique est indispensable » (Fichez et Benchenna, 2005, p. 50). La création du RUCA (Réseau des Centres d'Autoformation) ${ }^{1}$ procédait du même souci dès 1986. Mais, l'autorité publique, avec les campus numériques, introduit un élément nouveau dans les logiques économiques existantes, à savoir la capacité d'autofinancement au bout de trois ans, les subventions n'étant là au départ que pour aider aux investissements initiaux et les projets n'étant susceptibles d'être retenus que si la justification était faite de leur viabilité sur 3 à 5 ans.

L'analyse des terrains permet de constater que les concrétisations en termes d'universités virtuelles ou de campus numériques ne sont pas univoques, et en particulier les choix des acteurs sont fortement contraints par d'autres logiques que la pédagogie. Dans tous les campus numériques, l'opérateur, quel qu'il soit (CNED, UQAM, CCS ${ }^{2}$, consortium d'établissements, etc.), cherche à centraliser les offres de manière à pouvoir assurer la mise en relation avec la demande. Cet objectif permet de marier une (ou des) logique(s) pédagogique(s) portée(s) par les acteurs de terrains à une logique gestionnaire inédite. La 
technologie devient alors le vecteur de ce processus et porte une (ou des) logique(s) de rationalisation.

Ce constat nous a conduit à formuler trois hypothèses.

5 La première prend en compte le fait que les acteurs, dans la plupart des projets universitaires, sont amenés à intégrer les questionnements soulevés par les expériences précédentes menées dans leur secteur professionnel ou disciplinaire. De ce fait, les campus numériques ne nous semblent marquer qu'un moment particulier du temps de l'innovation qui procède d'un temps plus long. Nous supposons que ces campus représentent des expérimentations ayant pour objectif, soit de débloquer des facteurs politiques ou organisationnels nécessaires au passage à un système universitaire virtuel à venir, soit d'appréhender les problèmes qu'un tel système posera et les coûts qu'il demandera.

Ce phénomène d'apprentissage est typique de processus d'innovation d'envergure qui se déploient sur de très longues durées. Ces campus ne sont pas le point de départ du processus de mutation, ils sont eux-mêmes un moment dans une longue progression de projets expérimentaux et constituent en cela une nouvelle vague d'expérimentation dans un cycle d'innovation plus large où se testent des formes socio-organisationnelles inédites (Fichez, 2006).

7 L'hypothèse suivante concerne la gestion conflictuelle d'une double tension : la première est relative aux conflits existant entre les logiques d'origine et les objectifs affichés dans les appels à projets, et la seconde est liée au rapprochement nécessaire mais problématique entre deux stratégies : celle des acteurs assurant l'enseignement et celle propre aux chargés de la gestion du système universitaire. Les formes de production et de distribution créées dans chaque projet trouvent leurs origines, à la fois dans des logiques diverses liées au processus d'expérimentation lui-même, mais aussi dans les configurations d'objectifs pédagogiques et économiques propres à chaque type de formation ${ }^{3}$ et dans le consensus trouvé, ou non, entre les impératifs pédagogiques et gestionnaires. L'objectif poursuivi réclame l'adéquation entre le souci de généralisation des dispositifs porté par des gestionnaires, qui ont intérêt à la mise en place de formes de rationalisation nouvelles, et les impératifs pédagogiques premiers des enseignants.

La coexistence de logiques pédagogiques et économiques explique pourquoi l'analyse des campus numériques fait ressortir différents types de stratégies d'innovation qui se situent entre deux pôles.

Une première approche se fonde sur la "problématisation " qui a comme objectif la modernisation du système universitaire à travers la réponse à des besoins de terrain déjà articulés entre eux dans une filière spécifique. Alors qu'une seconde approche se fonde sur des activités d'uniformisation. Cette dernière stratégie cherche à standardiser des maillons spécifiques de la chaîne éducative $e^{4}$.

10 Si ces stratégies semblent s'exclure mutuellement, notre troisième hypothèse postule qu'elles peuvent se compléter dans un campus virtuel, les acteurs visant la mise en place de logiques industrielles inédites à travers l'introduction des réseaux dans le monde universitaire.

11 Les campus numériques présentant une grande diversité de développements, nous avons décidé de centrer notre analyse sur trois types de configurations qui renvoient chacune à des enjeux différents. Le premier cas, CampusCultura, est la résultante d'une stratégie de problématisation et d'une stratégie d'uniformisation. Autrement-dit, le projet, répondant 
à un diplôme, est fondé à l'origine sur l'analyse de besoins concrets et le projet de standardisation s'est greffé ensuite. À l'inverse, le second cas, C@mpuSciences, prolongement du projet de l'Université en Ligne créé par le RUCA à partir de 1995, s'appuie sur une tentative d'uniformisation des ressources en rupture avec les procédés de travail universitaire du moment qui n'a pas rencontré d'usages et vise donc à inverser le processus premier. Si dans le dernier cas analysé, canadien, les références et valeurs données à l'éducation sont quelque peu différentes de celles de la France, le stade de concrétisation y est plus engagé, rendant la comparaison du cas de Ryerson utile. D'une part, elle montre en effet l'importance des stratégies de réformes éducatives menées par le gouvernement provincial en charge de ce secteur et des transformations portées par les dispositifs technologiques au niveau du système central de l'université pour modifier les modes de fonctionnement organisationnel antérieurs ${ }^{5}$. D'autre part, elle permet de comprendre qu'un consensus peut être trouvé entre les différents niveaux du dispositif éducatif si une bonne marge de manœuvre est accordée aux enseignants sur les plans pédagogique et d'organisation des filières. Il semble de fait que le compromis trouvé par Ryerson après maints essais facilite la constitution d'une offre virtuelle qui fonctionne.

12 La première partie de cet article positionnera les différences des processus expérimentaux analysés et les débats soulevés par leur mise en œuvre respective. La seconde partie situera différents points de focalisation concernant les modes de production, les tensions entre des formes d'ingénierie contradictoires, les questions posées par l'accompagnement de l'étudiant et enfin la fonction d'intermédiaire entre offre et demande, qui obligent les acteurs à explorer des voies médianes entre des solutions extrêmes.

\section{Points de repères dans la généalogie des projets}

Les trois projets différents analysés ne trouvent pas leur origine dans les appels d'offre des campus numériques. Comme l'explique E. Fichez (2006), ces campus s'inscrivent dans des trajectoires de politiques, d'institutions et d'acteurs, entamées antérieurement. L'analyse de la genèse de ces projets sur le long terme montre de nombreux conflits liés à la divergence de points de vue entre les acteurs. Partant de présupposés différents et devant le plus souvent s'allier soit dans le cadre de l'appel à proposition, soit dans le cadre d'un établissement cherchant une nouvelle forme de cohérence d'ensemble, il leur est difficile de trouver un terrain d'entente commun. Seule une approche généalogique en profondeur permet de connaître les fils complexes qui se sont noués dans le temps et de mieux situer la complexité de ces processus ${ }^{6}$.

\section{Le cas CampusCultura : le résultat des « old boys » club de la culture}

14 Ce campus est initié par des professionnels de la médiation culturelle en association avec des universitaires. Cette alliance entre plusieurs institutions (CNED, universités, associations professionnelles du secteur culturel) précède de loin la création du campus numérique. En effet, dès les années 1990, ces acteurs tentent « d'allier le développement de formations dans les différents cycles universitaires pour les publics étudiants et celles à destination des publics non étudiants, celui des emplois-jeunes ayant constitué un mobile particulièrement déterminant pour la création en 1999 d'une licence à distance » (Benchenna et Fichez, 2005, 
p. 26). Cette démarche permet aux acteurs d'être en prise avec les besoins du terrain, le campus numérique devenant donc un moyen de moderniser un cursus préexistant et d'élargir son utilisation pour répondre aux besoins d'un public spécifique.

La concrétisation rapide est due à « deux facteurs (qui) ont rendu possible la mise en ligne des différentes unités d'enseignement constituant la licence en un temps record: la disponibilité d'une partie des contenus, sous forme de fichiers numériques et l'expérience acquise pour tous les cours dans les formations en présence, ainsi que le développement de la plate-forme CybEO par une société privée partenaire » (Benchenna et Fichez, 2005, p. 13). Cependant, dans sa phase d'élaboration, deux perspectives s'esquissent: un dispositif sur mesure formaté et homogénéisé d'une part, un dispositif sur mesure également, mais souple et respectant le style de chacun d'autre part.

E. Fichez et A. Benchenna commentent ainsi le raisonnement du directeur de la société privée CybEOsphere ${ }^{7}$, membre du consortium et tenant de la première perspective (2005, p. 33): "les offres de formation en ligne sont des objets économiques. Quand on décide de moderniser une formation, cela coûte cher et rentre dans un processus économique. C'est donc toute une logique industrielle qu'il faut mettre en œuvre. Il faut aussi un management motivé pour mettre en place ces solutions industrielles ». L'entreprise et 0 . Coppey, chef de projet pour le CNED, souhaitent contribuer à ce "management motivé " en fixant de nouveaux objectifs au cœur de CampusCultura : "Outre la commercialisation de la plate-forme, elle se positionne comme un prestataire de services agissant à la fois dans le domaine de l'ingénierie de formation et de l'ingénierie pédagogique, avec deux mots d'ordre: maitrise des coûts et offre de formation sur mesure » (idem, p. 16). Ainsi, sur les objectifs de modernisation propres à une filière de formation continue, viennent se greffer pour le directeur de la société des objectifs d'ingénierie, avec l'idée d'un transfert commercial possible du modèle expérimenté. Mais, sa perspective ne sera pas retenue et c'est plutôt à la seconde, celle des professionnels de l'enseignement, qu'il se ralliera : «celle-ci privilégie la mutualisation des questions nées de l'expérience et des réponses apportées pour en faire un 'bien commun' et s'efforce d'en faire un processus continu permettant d'affiner et de capitaliser les acquis pour en tirer parti dans la suite du projet » (Fichez et Benchenna, id., p. 36).

\section{De l'Uel à C@mpusciences ou l'impossible cheminement vers le sur mesure $^{8}$}

17 La notion de "sur mesure » mise en avant par les acteurs de CybEOsphere se retrouve dans le cadre du projet de l'UeL. L. Petit (à paraitre, 2008) attire l'attention sur les germes de conflit que cette notion produit : " La notion d'enseignement sur mesure, très générale, dans laquelle pouvaient se retrouver de nombreux courants de pensée, des approches pédagogiques distinctes voire contradictoires, des réseaux porteurs d'intérêts variés (ceux des centres de téléenseignement comme des centres d'autoformation, ceux des informaticiens comme des audiovisualistes, etc.) avait, à cette époque, un pouvoir fédérateur réel ». Mais des points litigieux ont divisé les membres du Ruca à propos de la mise en œuvre du projet d'enseignement sur mesure: ils ont fait le choix de privilégier la production d'une ressource linéaire et ceci, curieusement, au moment où l'idée du "sur mesure de masse " défendue par M. Quéré a pris beaucoup d'ampleur. Aux yeux de celle-ci, «le Ruca apparaît [pourtant] alors comme l'un des lieux privilégiés de la possible conciliation " affichée dans son rapport en 1994, à savoir "la volonté de concilier, via le sur mesure et le pilotage de l'offre pédagogique par la demande, deux exigences, jusqu'alors tenues peu ou prou pour contradictoires: 
la personnalisation de l'enseignement et l'industrialisation de la formation » (Combès, Moeglin, p. 16).

N'est-il pas contradictoire que le Ruca ait décidé, en 1996, la production de ressources se conformant à un programme et à « une présentation et une ergonomie identique » qui favorise la constitution d'une "véritable collection au sens éditorial du terme $"^{9}$ alors que Maryse Quéré prônait une rupture quant au fonctionnement du système éducatif, en attribuant à la demande un rôle déterminant par rapport à l'offre, et préconisait un déplacement radical du centre de gravité de la filière de l'amont vers l'aval ? Fallait-il penser au projet en termes de production de contenu ou en termes d'accès à une offre flexible, malléable et structurée à la demande ?

L'adoption d'un mode flexible et ouvert d'accès aux ressources proposé par l'université de Bordeaux dès $1991^{10}$ impliquait pour le Ruca de « modifier en profondeur des relations entre universités-partenaires en même temps que des transformations substantielles des manières d'enseigner et d'apprendre... » (Combès, Moeglin, 2005, p. 12). A l'époque, l'ouverture vers de nouvelles modalités éducatives inédites à instaurer dans des structures encore marquées par une organisation centralisée et hiérarchisée est vite apparu à la majorité des expérimentateurs comme un objectif difficilement atteignable, ce qui explique le choix de produire un ensemble de programmes fondé sur "une économie générale plus proche de la présentation du menu que celle de la carte» (idem). Cette production (qui deviendra l'Université en Ligne-UeL) ne rompait donc en rien avec la logique transmissive. Ainsi, le constat doit être fait que le Ruca a préféré « l'enseignement par l'amont à l'apprentissage par l'aval » (idem, p. 18), même si l'analyse de cet ensemble de ressources menée par L. Petit (2006) montre que coexistent en son sein la construction d'une base de données de type encyclopédique et l'amorce d'une approche modulaire mise en place par l'université de Bordeaux 1 au niveau local. La stratégie de rupture avec les procédés antérieurs, qui était au fondement du projet du Ruca, n'a donc pas été menée jusqu'au bout. Et en réalité, elle a conduit d'abord à une auto-production qui n'a engendré qu'une auto-consommation des ressources pédagogiques par leurs auteurs. La logique d'une production fondée sur la table des matières à enseigner s'est imposée par un souci d'utilisations multiples (FI/FC/ $\mathrm{EAD}$ ), au niveau national voire international, et a conduit à l'effet inverse de la formation sur mesure : les critères de malléabilité indispensable dans le cadre d'une offre flexible et structurée à la demande n'ayant pas été respectés, les enseignants - principaux intéressés visés - ne se sont pas sentis concernés. La mise à jour de ces faits explique le revirement ensuite vers les services que cherche à mettre en place C@mpuSciences en 2002. A ce campus numérique incombe donc de générer des usages en créant l'ingénierie de services qui favorisera cette souplesse, mais chacune des filières - formation initiale, continue et à distance - envisage la prestation à offrir de manière très différente. Ce sont donc plusieurs projets qui cohabitent pour l'instant (Combès, Moeglin, 2005).

\section{Le cas canadien : la création d'un environnement virtuel commun à toutes les filières}

20 Au Canada, la normalisation de pratiques médiatisées dans tous les domaines de la vie commence à s'étendre, l'école étant un vecteur d'incitation à ce changement. Ainsi, les étudiants canadiens ont de plus en plus tendance à s'inscrire simultanément dans les formations initiale, continue, et à distance dans le but de poursuivre des formations accélérées ${ }^{11}$. 
21 Les universités tentent de formaliser l'instauration de ces parcours accélérés à travers des projets qui modifient les modes de fonctionnement universitaire en profondeur. Au fil du temps, elles utilisent plusieurs stratégies dans la mise en œuvre d'un campus virtuel. Durant les années 1990, les administrateurs proposent des réformes en rupture avec les règles de l'époque. Les tentatives de changement s'adressent au système dans sa totalité et cherchent à y introduire le mode de management des entreprises privées. Mais au-delà, il s'agit aussi de faire adopter un ensemble de valeurs fondé sur l'évaluation du succès d'une filière en fonction de critères économiques et non plus pédagogiques. Les réformes annoncées impliquent également de nouvelles normes de travail pour le corps enseignant, marquées par l'introduction d'un système de production propre au matériel éducatif marchand et de celui de la formation continue. Bien évidemment, une telle proposition rencontre une résistance importante de la part des enseignants de la formation initiale qui refusent de laisser de tels modèles supplanter les leurs. Tel est le cas à l'université de York qui connaît en 1995 d'importantes grèves générales qui mettent fin à ces initiatives.

Les années 2000 connaissent un changement important des politiques menées. À la différence des tentatives conduites dans les années 90, les instances universitaires ne cherchent plus à transformer le dispositif entier. Chaque pôle utilise sa propre approche en créant un système hybride où de nouveaux modèles éducatifs viennent se greffer sur les modèles traditionnels. Chacun développe des tactiques internes adaptées à son contexte spécifique. Rappelons que chaque université en Ontario ${ }^{12}$ est un organisme autonome qui conçoit et gère sa politique d'établissement.

23 A Ryerson, les tentatives de réforme actuelles ne s'effectuent pas à travers des projets expérimentaux, mais par l'intégration des TIC dans les usages existants. L'intention est de laisser le terrain, autrement dit le corps enseignant, définir progressivement de nouveaux usages qui correspondent à ses besoins. Pour ce faire, l'université introduit des outils de travail virtuels et un portail «my.ryerson.ca». Ce portail agrégateur des usages infocommunicationnels permet la mise en commun des offres tout en s'inscrivant dans la continuité du régime d'enseignement actuel. Comme nous le verrons, sont ainsi générées de nouvelles formes d'ingénierie pédagogique et de formations hybrides qui allient les processus de production des formations continue et à distance aux processus de médiation de la formation initiale. Dans le long terme, l'université constituerait le point d'accès aux ressources variées offertes par ces différentes filières, leur lieu d'origine devenant transparent aux usagers.

À travers la genèse des projets, nous remarquons que les différentes logiques des acteurs donnent naissance à des solutions distinctes qui trouvent leur ancrage dans la longue suite de projets et tentatives de nature disparate. Il convient maintenant dans une approche plus synchronique de repérer quelques points litigieux rencontrés, le plus souvent, par chacun des campus.

\section{Des modes de production et de diffusion confrontés aux enjeux institutionnels et organisationnels}

Dans les discours concernant chacun des campus, on repère la volonté d'instaurer des processus de production des ressources plus ou moins rationalisés et standardisés. Par exemple, dans le cas de CampusCultura, l'acteur industriel (CybEOsphere) envisage au 
départ: " de rechercher comment automatiser un processus de production de cours grâce à des logiciels en vue d'économies d'échelle dans la production " (Fichez et Benchenna, 2005, p. 35). L'objectif aurait été la mise en œuvre d'une «normalisation des cadres d'écriture » où les auteurs se seraient vu imposer des méthodes, des règles vis-à-vis des cahiers de développement, une certaine modélisation des tâches par la mise en place d'un outilauteur.

Les développeurs de l'Uel comme nous l'avons souligné, devaient se conformer à « une présentation et une ergonomie identique de façon à constituer une véritable collection au sens éditorial du terme... ainsi qu'à l'adoption d'une charte graphique et d'une maquette commune " imposées par le Ministère (Petit, 2008). A Ryerson, la formation à distance a envisagé " l'instauration de processus de formatage standardisé de production, de structuration et de présentation des contenus pour réduire le temps dévolu au développement du cours et le temps consacré par l'étudiant à la recherche des contenus dont il a besoin » (Bal, 2005, p. 31).

Mais qu'advient-il de ces objectifs normalisateurs émanant d'acteurs autres que les enseignants, dans la réalité?

\section{La production de contenu questionnée}

Dans le cas de CampusCultura, ils relèvent d'un vœu pieux, tout ceci étant difficile à imposer aux enseignants non parti-prenantes des processus expérimentaux. A Ryerson, des activités de production et d'ingénierie de formation peuvent être redéfinies et assignées à d'autres acteurs engagés par l'université, mais les nouveaux agents ne peuvent pas être imposés au corps enseignant. Ainsi, les responsables de la formation à distance doivent, à contrecœur, conserver au professeur un rôle central dans le processus de production car il est le seul à pouvoir légitimement intervenir sur le contenu, empêchant ainsi les spécialistes de l'enseignement médiatisé d'intervenir à ce niveau. D'où le recours à un formatage de structure et à des parcours de cours standardisés, mis en place par l'équipe de production, ce qui lui permet de contrôler la présentation des contenus. Dans le cas de l'UeL, les objectifs ont été réinterprétés par les enseignantsinnovateurs, conduisant au sein du même produit à la cohabitation de modèles de développement différenciés : l'un majoritaire favorise la linéarité et donc un modèle de production de contenu, l'autre préserve une certaine modularité ${ }^{13}$ en phase avec la formation à la demande $\mathrm{e}^{14}$. Ainsi «à un processus d'éditorialisation de cours qui aboutit à des réalisations allant du polycopié plus ou moins enrichi au manuel numérique s'oppose dans l'UeL un processus d'agencement de ressources autonomes plus ou moins standardisées, susceptibles d'être réagencées autrement et dans d'autres contextes » (Petit, 2008).

La standardisation des processus de production de contenu qui suppose une certaine soumission des enseignants à des règles industrielles n'apparait donc pas évidente au vu de la réalité.

\section{L'indexation : une solution pour concilier deux formes d'ingénierie?}

Aux problèmes concernant la production s'ajoute la tension entre deux formes d'ingénierie, particulièrement visibles au sein de C@mpuSciences et à Ryerson. Si l'une est relative à la production de contenu se conformant à un programme a priori, l'autre tente de privilégier une ingénierie du service éducatif ou ingénierie de médiation qui renvoie aux procédures de standardisation voire de normalisation de ressources outillées, 
soit pour la mise en forme de ressources (en vue de leur modularisation et de la gestion des trajectoires), soit pour l'uniformatisation des outils de travail virtuels (la standardisation passant à Ryerson par la plate-forme, ainsi que par l'évolution de procédés administratifs et juridiques).

Cette tension nous renvoie à l'interrogation suivante: de quelle manière s'effectue l'articulation de la production et de la diffusion dans les campus analysés? Pour CampusCultura cet aspect ne s'est pas avéré un problème à résoudre, étant donné que l'innovation est cantonnée à une filière de formation très circonscrite où producteurs et diffuseurs sont encore étroitement liés. Par contre, on peut s'interroger sur la manière dont C@mpuSciences a envisagé cet objectif, qui lui fut assigné au départ, et les raisons qui l'ont poussé, ainsi que l'université de Ryerson, à privilégier comme solution, en priorité, l'indexation. Celle-ci permet d'établir une description du contenu à l'aide d'un langage documentaire et intervient donc tant par rapport aux modes de production que par rapport à ceux de la distribution et circulation des ressources.

Dans C@mpuSciences, l'indexation « ne pose pas de problème particulier, ni pour les tenants de l'UeL qui y voient un moyen de faciliter les entrées transversales dans les contenus numérisés, $n i$ aux partisans d'une industrialisation de l'ingénierie, qui y voient un palliatif de la modularisation » (Combès, Moeglin, 2005, p. 41). Cependant le choix d'indexer les chapitres ne permet pas d'aboutir à la solution idéale qui permettrait un repérage, puis un découpage de la ressource en petites unités autosuffisantes ${ }^{15}$, jusqu'à arriver à une situation où l'enseignant identifierait, grâce à un moteur de recherche, les unités les plus fines qui l'intéressent et qu'il mettrait dans une "coquille» de cours. Faciliter l'accès modulaire aux contenus numérisés de l'UEL reste donc encore le principal défi auquel c@mpuSciences est confronté et sur lequel ses membres se divisent le plus.

Ryerson a cherché à standardiser et à centraliser les outils de travail virtuels plutôt que de produire des contenus. Ceci permet de référencer des ressources préexistantes, non standardisées ni découpées, qui restent sur les serveurs des propriétaires. Cette solution, qui permet de contourner les problèmes de droits d'auteurs et juridiques, évite d'aborder, là aussi, les questions de modularisation. Ces procédés d'indexation ne favorisent pas une formation à la carte, mais l'inclusion, au delà des ressources de contenu, des services et des programmes non pédagogiques dans l'offre. Ceci dans le but de construire des offres élargies participant à la création d'un guichet unique, donnant un accès standardisé aux services et opérations administratives, pédagogiques et commerciales des institutions universitaires. Il s'agit donc d'une option qui correspond bien à la volonté d'uniformiser des outils de travail virtuel.

Dans tous les cas, on relève, comme l'avaient repéré Y. Combès et P. Moeglin (2005, p. 35), qu'au modèle de la production lourde, prenant du temps mais fournissant des contenus numérisés appelés à durer, devrait se substituer une production plus légère ${ }^{16}$, sollicitant d'autres outils, et dès lors mieux en phase avec les évolutions et les modulations de la demande.

\section{L'enjeu de la médiation tutorale dans le cadre d'une virtualisation de l'enseignement}

Nous abordons sous ce thème la façon dont sont pensées les formes d'encadrement et leurs modalités dans les campus étudiés. C@mpuSciences s'engage dans deux modalités 
complémentaires : la médiatisation (tutorat en ligne) et l'objectivation (tutorat assuré par une plate-forme). Si la première option conduit à la division industrielle du travail entre enseignants et tuteurs, la seconde, qui s'esquisse, est de proposer une réponse automatisée au problème posé par l'étudiant: elle viserait, à terme, à repérer les questions récurrentes des étudiants pour apporter l'explication complémentaire, favorisant le rapprochement de l'acte de production et de l'acte de formation.

$\mathrm{Au}$ sein de CampusCultura, au-delà des tutorats mis en place, «le forum est un espace de communication permettant la mise en commun des expériences individuelles d'apprentissage " (Fichez et Benchenna, 2005, p. 55). S'il n'existe pas de formes de rationalisation de ce type d'activités pour l'instant, les auteurs de l'étude "Usages et usagers $»^{17}$ concernant ce campus recommandent d'aller "dans le sens d'un tutorat encore plus intensif et plus individualisé $"^{18}$ (idem, p. 20).

Les formes de self-service adoptées à Ryerson, qui s'établissent au niveau de l'utilisation des ressources, s'accompagnent également d'un encadrement virtuel humain. L'établissement de la plate-forme unique, imposée à tous les secteurs, favorise l'implémentation de nouveaux modes d'ingénierie de formation fondés sur des services institutionnels de production et d'encadrement des étudiants propres à une offre virtuelle. Les changements significatifs concernent le rôle des tuteurs, un groupe de professionnels qui vient d'être syndicalisé, ce qui facilite la mise en œuvre de normes de travail et permet, à la fois, de définir ce que recouvre cette fonction et de l'instaurer comme un métier à part entière ${ }^{19}$.

\section{Entre campus numérique intégrateur ou courtage : les incertitudes du modèle à inventer}

Diverses questions majeures restent en suspens, concernant la place à réserver aux contenus existants, le fonctionnement pédagogique, l'organisation de la formation et plus précisément le fonctionnement et le statut à donner à ces campus quant ils concernent plusieurs types de formation.

Deux principes contradictoires semblent présider aux agencements organisationnels de c@mpuSciences et de Ryerson. Le premier est celui de mutualisation qui suppose le maintien, voire le renforcement, de l'hétérogénéité institutionnelle et fonctionnelle des partenaires (internes ou externes), le second est celui de synergie qui renvoie à un idéal collaboratif, associant des acteurs au sein d'une seule et même structure, aussi homogène que possible. Pour le dire autrement, ce qui est en jeu ici n'est autre que l'alternative fédération/union. Devant cette alternative, chacun des deux campus hésite à prendre une direction définitive. Les acteurs de C@mpuSciences affirment se rallier à l'idée du changement, mais en fait, ils n'abandonnent pas tout à fait le principe de la continuité : à la synergie, qu'ils essaient de mettre en œuvre en créant une marque commune, ils préfèrent encore parfois la mutualisation (chaque filière, FI, FC, EAD, développant son propre projet $)^{20}$; de même, par rapport à l'invention de services, voie qu'ils privilégient, ils n'abandonnent pas tout à fait certaines activités de production. A l'identique, l'université de Ryerson cherche un juste milieu: elle vise à travers la plate-forme le principe de synergie, en instaurant un processus de centralisation de management des services, et applique le principe de mutualisation vis-à-vis des ressources et des cours. L'intérêt devient de faire coexister différents usages sans les forcer à s'amalgamer car leur coexistence dans le système de distribution crée une marque virtuelle commune 
pour Ryerson. C'est pourquoi la fédération paraît une démarche plus appropriée même si elle contredit les idéologies de départ. Cette semi-institutionnalisation (Combès, Moeglin, 2005, p. 31) correspond, dans les deux cas, à une troisième voie entre une organisation centralisée et une dimension coopérative: la volonté de management minimal est destinée à laisser le champ libre aux initiatives singulières et procède plus par régulation que par direction.

Pour illustrer cette réflexion et la prolonger, il nous paraît important d'évoquer deux formes organisationnelles: d'une part, celle duprojet Formation Sur Mesure (FSM) développée au sein de C@mpuSciences, qui apparaît comme l'une des voies possibles, destinée à la formation continue et qui consiste en l'organisation du courtage et d'une logique de service ; d'autre part, celle du campus intégrateur, propre à Ryerson, tournée vers le self-service généralisé.

41 En ce qui concerne FSM, l'objectif vise à agréger contenus numérisés, services d'accompagnement et étudiants appartenant à diverses universités, étant donné l'insuffisance de l'offre et de la demande locales en formation continue. Pour ce faire doivent s'établir des conventions entre l'université d'origine qui inscrit et suit l'étudiant, celle effectuant le courtage et celle fournissant les contenus. Ainsi l'ensemble de la filière doit être réorganisée par le courtier, intermédiaire entre universités demandeuses et universités offreuses. Ce projet, fondé sur « un modèle de gestion de projet où l'on décline quelles sont les responsabilités et les sujets sur lesquels portent les responsabilités ${ }^{21}$ est l'anti-modèle industriel qu'au titre de C@mpuSciences les membres de la formation continue opposent au modèle de l'UEL, puisqu'il cherche à adapter a posteriori certains contenus à certains publics dans une optique de « sur mesure de masse ». Il est cependant loin de faire l'unanimité, certains membres du consortium craignant d'être transformés en sous-traitants en amont comme en aval, privés de la fonction stratégique du courtier. De plus, la formalisation d'un tel projet implique encore de clarifier les conditions financières et de viabilité du système ainsi que les questions de responsabilité juridique ${ }^{22}$.

En ce qui concerne Ryerson, les questions de fond posées précédemment concernant la nature des ressources produites, l'articulation de modes de fonctionnement différenciés à l'amont et à l'aval, la détermination du rôle de chacun, la division du travail, la formation des usages, sont éludées au profit de la mise en avant de la légitimité institutionnelle de l'université. Le choix est de faciliter la mise en œuvre de projets variés qui faciliteront l'instauration d'un campus intégrateur. Celui-ci se fonderait sur les approches diversifiées des enseignants pour l'élaboration d'une offre globale, multiple et ouverte, à travers une plate-forme technologique. "Cette plate-forme a valeur d'expérimentation pédagogique et structurelle car elle va permettre à tous les acteurs de tester le développement de nouvelles formes pédagogiques ainsi que différents types de processus de flexibilisation des prestations éducatives et de fédération des formations initiale, continue et à distance» (Bal, 2006). Le cas de Ryerson s'inscrit ainsi dans une dynamique d'établissement et non dans une logique interuniversitaire, car les dangers de perte de monopole sur l'offre pour chaque université paraissent trop grands ${ }^{23}$. L'objectif est de laisser les pratiques évoluer en fonction des normes et des cultures locales tout en inscrivant les mutations dans un processus de construction de formes d'ingénierie de formation permettant l'hybridation des projets innovants des formations initiale, continue et à distance, ceci sous-entendant un déplacement des frontières juridico-administratives qui séparent ces trois filières.

Le souci d'établir un campus intégrateur conduit à privilégier les problèmes d'interopérabilité, de procédures collectives d'identification, de sélection, de 
hiérarchisation des ressources (menées par des pôles spécialisés informatique ou structure $\mathrm{EAD}$ ) laissant à chacune des filières le soin de choisir entre l'approche descendante ou ascendante par rapport à leurs publics. L'objectif est d'offrir un guichet unique fédérant les fonctions administratives, logistiques, enseignement et ingénierie de service, et incluant tant les services de tutorat et d'accompagnement de l'acte éducatif que les services bureaucratiques et d'accès à la bibliothèque, les services techniques, etc.). "L'administration n'annonce pas officiellement ses intentions vis-à-vis d'un système de self-service et de services éducatifs sur mesure qui viseraient des cibles non-universitaires, mais plusieurs éléments semblent aller dans ce sens " (Bal, 2006). L'importance de cette fonction centrale s'amplifie lorsque les offres prennent une envergure marchande. Ce qui explique pourquoi les universités canadiennes privilégient l'image de l'université, chaque établissement pouvant s'accorder légitimement ce rôle. L'université peut se muer, à terme, en agent courtier qui possède un avantage concurrentiel important dans la compétition grâce une offre variée (celles de la FI, de la FAD et de la FC) proposée à des publics divers (étudiants et entreprises), susceptible de répondre à des besoins diversifiés.

\section{Conclusion} contexte donne naissance à des formes d'ingénierie de production et de médiation spécifiques, et influence la décision d'aller vers des formes organisationnelles fondées sur le principe de la mutualisation ou de la synergie. Les projets servent à évaluer les problèmes et les coûts que de telles évolutions demandent. Les analyses menées mettent en valeur l'importance, plus ou moins grande selon les contextes, des facteurs économiques, politiques et organisationnels nécessaires au passage à un système universitaire virtuel et les conflits inhérents à ces changements. Reste à savoir si cette série d'innovations tend vers un réel changement de paradigme éducatif. L'avenir de cette innovation dépendrait alors moins des actions menées par les porteurs de projet que d'éléments relatifs à des changements sociaux plus profonds, comme le souligne dans ce même numéro P. Grevet.

Nous avons relevé des tendances polymorphes empruntant à diverses formes industrielles. Il semble toutefois que la résolution du problème posé par l'inéluctable conjonction d'une ingénierie de production, nécessaire mais insuffisante, et d'une ingénierie de médiation passe par une modalité organisationnelle inédite que l'on ne rencontre ni dans le champ de l'éducation et de la formation (sous ses formes traditionnelles), ni dans celui de l'édition. Actuellement sont perceptibles diverses tendances relatives à la flexibilisation des modes de fonctionnement et interactions pédagogiques, à la décentration de la relation enseignant-enseigné vers l'enseigné, à la division entre «base arrière » et « base avant » dans les dispositifs, à une industrialisation qui affecte moins l'amont de la chaîne pédagogique (conception, production) que l'aval (accès à des connaissances par l'usager), au primat de l'ingénierie de médiation par 
rapport à celle de production. Ce qui supposerait qu'on passe progressivement « d'innovations cantonnées jusqu'alors à une série de changements marginaux à une innovation instauratrice, au sens de M. de Certeau » (Combès, Moeglin, 2005, p. 12). Ceci reste encore une hypothèse à vérifier à l'avenir, qui, si elle se produit, prendra encore du temps. Mais la question du changement de paradigme éducatif oblige à rechercher la (ou les) cohérence(s) susceptible(s) de se dessiner.

\section{BIBLIOGRAPHIE}

Bal, A., (2006), Virtual University Education as an Agent of Social Change: Educational Design as a Battleground for Liberalist and Humanist Socio-economical Values, International Journal of Learning, Volume 12, Issue 2, pp. 115-122.

Bal, A., (2005), Virtual Higher Education: A Liberalist or Humanist Socialization Tool, International Journal of Technology, Knowledge and Society Volume 1, Issue 1, pp. 132-139.

Bal, A., (2003), Réflexions sur l'adaptation industrielle de la corporation du savoir à l'espace virtuel, Thèse de doctorat en sciences de l'Information et de la Communication, Université Paris 13, reproduite par Presses Universitaires/ANRT, Thèses à la carte, Lille.

Bal, A., (2005), Modèles économiques et enjeux organisationnels des Campus numériques : Le cas My.Ryerson.ca, accessible aux adresses indiquées à ERTe CN 2005.

Barbot, M. J. et Combès, Y., (2006-4), « Penser le changement de paradigme éducatif lié aux TIL », in : éducation Permanente, $\mathrm{n}^{\circ} 169$, Pédagogie et numérique. Contradictions ? Convergences?, pp. 133-152.

Combès, Y., (2008), « Produire et diffuser des ressources : entre industrialisation et marchandisation » chapitre 3, in : Delaunay-Jacquinot G. et Fichez E. (éds.), Université et TICE : chronique d'une innovation annoncée, De Boeck Université.

Combès, Y., (2001), Éditorialisation, partenariat, commercialisation, Rapport de la commission $n^{\circ} 1$, in : L'Université en Ligne - Premier Cycle Sur Mesure -, Les rencontres de La Rochelle le 5 et 6 juillet 2001, Actualités de l'université en ligne, http://www.uel.education.fr, pp. 10-14.

Combès, Y., (2000), L'appropriation des ressources pédagogiques par les enseignants, Rapport de la commission $n^{\circ} 4$, Les ressources scientifiques de l'Université en Ligne - Premier Cycle sur Mesure - : Quels usages, journées d'étude à Marseille le 29 et 30 juin 2000, Actualités de l'université en ligne, http:// www.uel.education.fr, pp. 25-31.

Combès, Y. et Moeglin, P., (2005), Modèles économiques et enjeux organisationnels des Campus numériques: C@mpuSciences : d'un modèle industriel à l'autre, accessible aux adresses indiquées à ERTe CN 2005.

Deceuninck, J., (2005), Modèles économiques et enjeux organisationnels des Campus numériques : campus Forse, accessible aux adresses indiquées à ERTe CN 2005.

ERTe CN, (2005), Résultats 2005 de l'ERTe (Équipe de Recherche Technologique éducation) « Modèles économiques et enjeux organisationnels des campus numériques ", accessible à www.ifresi.univ- 
lille1.fr/SITE/2_Recherche/22_Programmes/ERTe/ERTe.htm ou à partir de la page d'accueil www.mshparisnord.org/.

Fichez, E., (2006), «Campus numériques : des ambitions à l'épreuve des terrains ", Distances et Savoirs, vol. 4/3, Hermès-Lavoisier.

Fichez, E. et Benchenna, A., (2005), Modèles économiques et enjeux organisationnels des Campus numériques : le cas CampusCultura, accessible aux adresses indiquées à ERTe CN 2005.

Grevet, P., (2005), Modèles économiques et enjeux organisationnels des Campus numériques : L'expérience économico-institutionnelle de Canège, accessible aux adresses indiquées à ERTe CN 2005.

Horn, F. et Lamarche, T., (2005), Modèles économiques et enjeux organisationnels des Campus numériques: L'Université médicale virtuelle francophone, accessible aux adresses indiquées à ERTe CN 2005.

Petit, L., (2006), « La production de ressources numériques et la professionnalité des enseignantschercheurs, la cas de l'Université en ligne ", in : Éducation permanente, dossier Pédagogie et numérique, Contradictions? Convergences ?, $n^{\circ}$ 169, 2006-4, pp. 51-65.

Petit, L., (2008), « Université en ligne - Premier cycle sur mesure », retours sur un projet emblématique, chapitre 2, in : Delaunay-Jacquinot Geneviève et Fichez Elisabeth (éds.), Université et TICE : chronique d'une innovation annoncée, De Boeck Université.

Tremblay, G. et Fauteux, S., (2005), Modèles économiques et enjeux organisationnels des Campus numériques : le DESS en santé mentale de la Téluq, accessible aux adresses indiquées à ERTe CN 2005.

\section{NOTES}

1. Le RUCA créé en 1986 a permis de développer des ressources correspondant à plus de mille heures de formation en sciences (mathématiques, physique, chimie, biologie...) destinées aux deux premières années de DEUG. Ces ressources nommées «Université en Ligne » constituent la base de données de contenu sur laquelle s'appuie C@mpuSciences, qui s'est donné pour tâche de développer de services permettant de valoriser l'UeL dans des contextes différents (formation initiale, continue et à distance).

2. Communication and Computing Services: Services de communication et d'Informatique de Ryerson.

3. La nature des prestations et les procédés utilisés se définissent en fonction de l'habitus professionnel des acteurs, à savoir leur appartenance à la formation initiale, continue ou à distance, leur appartenance disciplinaire ou professionnelle, le public visé et l'envergure du projet : certains campus ne recouvrent qu'une filière très spécialisée (CampusCultura), d'autres plusieurs disciplines (c@mpusciences).

4. La problématisation privilégie l'adaptation au contexte, c'est-à-dire la mutualisation des questions nées de l'expérience et des réponses apportées pour en faire un «bien commun » et s'efforce d'en faire un processus continu permettant d'affiner et de capitaliser les acquis pour en tirer parti dans la suite du projet. La normalisation impose des règles, en rupture avec le fonctionnement antérieur, qui peuvent devenir artificielles et mal fondées pédagogiquement (Fichez, Benchenna, 2005, p. 35).

5. Voir à ce propos Bal, 2006.

6. Nous avons nous-mêmes suivi deux réalisations: A. Bal a suivi les projets technologiques de Ryerson et les a analysés dans sa thèse qui couvre la période 1995-1998 et dans des travaux récents de 2000 à 2006 ; Y. Combès a suivi les projets antérieurs à $\mathrm{C} @$ mpuSciences sur le long terme (Y. Combès, 2000, 2001, 2007 et Y. Combès, P. Moeglin, 2005). Quant à CampusCultura, nous 
avons dégagé de la monographie réalisée par E. Fichez et A. Benchenna (2005) quelques repères importants.

7. Le CNED a choisi cette société, CybEOsphere, qui appartient au monde expérimental de la recherche publique.

8. Pour une analyse plus détaillée des conflits et des enjeux, voir Combès, 2007 et Combès Moeglin, 2005.

9. Comme le précise le document intitulé «Vers un premier cycle sur mesure » (version du 23 juillet 1997).

10. Bordeaux a proposé aux membres du Ruca un programme qui ressemble à une plate-forme avant la lettre (concerto 813), et qui consistait à uniformiser les fonctions de suivi et d'orientation, à programmer les activités tutoriales, et qui visait la décomposition à terme de la chaîne éducative en segments séparés depuis la conception jusqu’à la réception en passant par la production, médiation, transmission (Combès, Moeglin, 2005, p. 15).

11. Par exemple, à Ryerson, les formations continue et à distance offrent le même cours quatre fois par année, tous les semestres, alors que la formation initiale ne les offre qu'une fois par an. Les étudiants s'inscrivent dans des cours d'été et substituent les crédits de la formation à distance ou de la formation continue à ceux de la formation initiale.

12. Cf. Bal, 2005, pour plus de détails.

13. La modularité pose deux problèmes de type différents. Dans un cas, celui de l'accès aux étudiants qui ont besoin d'une modularité de contenus pour pouvoir rompre avec la linéarité de l'enseignement qu'ils reçoivent. Dans l'autres cas, celui de l'aptitude des enseignants à prendre au sein des ressources de l'UeL les contenus qui les intéressent pour les réassembler à leur guise (Combès, Moeglin, 2005, p. 40).

14. $70 \%$ des ressources de contenu de l'UeL correspondent au modèle linéaire, $30 \%$ relèvent du modèle conservant en partie la philosophie du sur-mesure (Petit, 2006).

15. La définition des caractéristiques d'un grain de ressources pédagogiques est problématique. De plus il doit constituer une unité didactique minimale intégrant en cohérence un objectif, une tâche et une évaluation. Voir Barbot, Combès, 2006, p. 144.

16. Un modèle de production des contenus se dessine actuellement dans les filières de la formation initiale de Ryerson qui laisse une plus grande latitude à l'approche personnelle de chaque enseignant. Dans ce cas, il n'y a plus de standard qui leur soit imposé : ils peuvent créer leur propre équipe de production et définir les critères de standardisation pour la mise en œuvre des contenus ainsi que pour les processus pédagogiques.

17. Pouts-Lajus S. et Leccia E. (septembre 2004), « Évaluation formative accompagnant la mise en place de CampusCultura (CNED). Usages et usagers », Éducation et Territoires, 35 pages.

18. "Voir par exemple leur recommandation - intéressante, mais coûteuse en temps - concernant les aménagements à apporter au forum: il s'agirait de faire gérer par les tuteurs des listes de diffusion qui permettraient aux étudiants qui ne se connectent pas sur le forum de recevoir une sélection des messages utiles directement dans leur messagerie. On notera par ailleurs dans cette étude des analyses très intéressantes concernant la part importante prise par les étudiants dans la mise en commun de leurs expériences individuelles d'apprentissage, fonction complémentaire de celle exercée par les tuteurs " (Fichez, Benchenna, 2005).

19. Pour des prolongements sur cette question, voir Bal (2006).

20. Par exemple, les centres d'enseignement universitaire en Sciences n'ont aucun intérêt à une baisse des étudiants inscrits au profit d'un consortium ; aussi ne veulent-ils pas intégrer une offre commune car ils sont clairement en concurrence avec les acteurs de FC et FI.

21. Parole de l'acteur qui défend le courtage au sein de C@mpuSciences, repris dans Combès, Moeglin, 2005, p. 38.

22. L'avènement des futures universités thématiques ou celui des universités numériques en région pourrait constituer une étape sur cette voie. 
23. La structure du dispositif canadien empêche une logique inter-universitaire. Rappelons que le système universitaire canadien ne représente pas une structure éducative nationale. Les universités canadiennes sont en compétition financière les unes avec les autres car elles cherchent à attirer les meilleurs étudiants afin de s'assurer de l'obtention de bourses et de crédits de recherche supplémentaires. Cf. Bal, 2005 pour plus de détails.

\section{RÉSUMÉS}

Les auteurs partent de l'hypothèse que les campus numériques marquent un moment particulier du temps long de l'innovation. Les fluctuations de leurs orientations peuvent être lues comme la recherche de consensus entre les impératifs pédagogiques et gestionnaires et de solutions pour débloquer des facteurs politiques ou organisationnels nécessaires à l'évolution du système universitaire. La recherche d'une voie du juste milieu peut conduire à croiser les différentes logiques socio-économiques et les formes industrielles coexistant dans une organisation centralisée. Ceci en fédérant les fonctions administratives, logistiques, d'enseignement et d'ingénierie de services, et en introduisant une dimension coopérative pour la production de ressources.

This article is based on the hypothesis that emerging virtual campuses represent a specific phase within much longer innovation frameworks. The fluctuations within their directions can be understood as the quest for a consensus between pedagogical and administrative imperatives, and, for solutions that resolve political and organizational elements needed to introduce change within the current university system. The quest for a new solution that does not favor a logic over another can lead to evolutionist and ad hoc strategies which cross pollinate various socioeconomical logics, and their corresponding industrial forms, within a centralized organization. This outcome can be achieved via the aggregation and federation of multiple actors' administrative, logistical, pedagogical activities as well as services.

\section{INDEX}

Mots-clés : campus numérique, innovation, pédagogie, enseignement supérieur, modèle socioéconomique, management éducatif

Keywords : virtual campus, innovation, pedagogy, higher education, socio-economic model, educational management

\section{AUTEURS}

\section{ALEXANDRA BAL}

Alexandra Bal est assistante/professeur de l'Université Ryerson et chercheure associée à la MSH

- Paris Nord sur l'axe de recherche des industries de la culture et des arts. Ses travaux de recherche portent sur l'introduction des technologies de l'information et de la communication dans l'éducation universitaire de l'Ontario ainsi que sur le phénomène spécifique 
d'interdisciplinarité en jeu dans la création d'écologies médiatiques pédagogiques qui combinent les environnements virtuels et présentiels. Adresse électronique : abal@ryerson.ca.

\section{YOLANDE COMBÈS}

Yolande Combès est professeur à l'Université Paris 13, attachée au laboratoire LabSIC et à la MSH Paris Nord où elle est co-responsable du thème « socio-économie de la culture et de la communication » et membre du Séminaire Industrialisation de la Formation. Ses travaux de recherche portent sur les enjeux socio-économiques et organisationnels dans les industries culturelles et dans l'éducation. Elle a publié des études issues de ces terrains de recherche, en particulier sur l'enseignement supérieur et le champ de la formation en entreprise, et co-dirige avec $\mathrm{P}$. Bouquillion un ouvrage à paraître prochainement : Les industries de la culture et de la communication en mutation aux Presses Universitaires de Vincennes. Adresse électronique : yolande.combes@wanadoo.fr. 